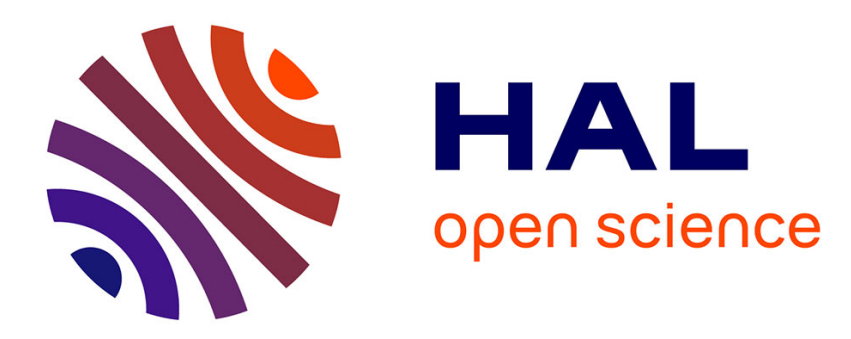

\title{
Étude et réalisation d'un interféromètre hertzien à trois ondes de grande sensibilité
}

\author{
R. Sardos
}

\section{To cite this version:}

R. Sardos. Étude et réalisation d'un interféromètre hertzien à trois ondes de grande sensibilité. Revue de Physique Appliquée, 1969, 4 (1), pp.29-32. 10.1051/rphysap:019690040102900 . jpa-00242885

\section{HAL Id: jpa-00242885 https://hal.science/jpa-00242885}

Submitted on 1 Jan 1969

HAL is a multi-disciplinary open access archive for the deposit and dissemination of scientific research documents, whether they are published or not. The documents may come from teaching and research institutions in France or abroad, or from public or private research centers.
L'archive ouverte pluridisciplinaire HAL, est destinée au dépôt et à la diffusion de documents scientifiques de niveau recherche, publiés ou non, émanant des établissements d'enseignement et de recherche français ou étrangers, des laboratoires publics ou privés. 


\title{
ÉTUDE ET RÉALISATION D'UN INTERFÉROMÈTRE HERTZIEN A TROIS ONDES DE GRANDE SENSIBILITÉ
}

\author{
Par R. SARDOS, \\ Laboratoire d'Optique Ultra-Hertzienne (E.R.A., G.N.R.S.) et Laboratoire de Physique Expérimentale,
}

Faculté des Sciences de Bordeaux.

(Reçu le 16 octobre 1968.)

\begin{abstract}
Résumé. - L'auteur donne le principe d'un interféromètre de précision à trois ondes inspiré des procédés de détection de phase permettant la mesure et l'enregistrement des très faibles déphasages et atténuations. Il en décrit une réalisation pratique pour la longueur d'onde de $3 \mathrm{~cm}$.
\end{abstract}

Abstract. - A precise interferometer with three branches is described. It is inspired from phase-lock techniques and allows measurements or recording of very weak changes in phase and amplitude. The experimental set-up is described for X-band.

Les interféromètres hertziens ont été généralement inspirés des modèles optiques et de nombreux interféromètres à deux ondes ont vu le jour. Mais par suite de considérations techniques, il est très difficile dans les interféromètres micro-ondes de mesurer les faibles variations de phase accompagnées de faibles variations d'amplitude. En effet, les atténuateurs ont une sensibilité limitée et introduisent toujours une faible variation de phase, tandis que les déphaseurs étalonnés, de sensibilité limitée également, introduisent une faible variation d'amplitude. Pour s'affranchir en partie de ces inconvénients, diverses solutions ont été proposées $[1,2,3,4$, etc. $]$, mais il n'en demeure pas moins qu'il est encore difficile de faire des mesures de faibles variations de phase et d'amplitude et surtout d'en faire l'enregistrement. C'est pourquoi, poursuivant diverses études d'optique hertzienne, j'ai été conduit à étudier un interféromètre non usité en optique mettant en jeu les ressources de la détection synchrone. Cet interféromètre est un interféromètre à trois ondes. Compte tenu du matériel dont dispose le Laboratoire, il n'a pu être réalisé qu'à la longueur d'onde de $3 \mathrm{~cm}$, mais il peut facilement être réalisé dans tout le domaine des micro-ondes, car il ne fait appel qu'à des éléments classiques.

Pour faciliter l'étude de l'interféromètre à trois ondes, rappelons brièvement quelques résultats utiles concernant les interféromètres à deux ondes.

Dans un interféromètre à deux ondes, on fait interférer deux faisceaux issus d'une même source. Une partie au moins des ondes se propageant dans des guides, on peut représenter les vibrations qui interfèrent sous la forme :

$$
E_{1}=A_{1} \exp \left[j\left(\omega t-\varphi_{1}-\frac{2 \pi d_{1}}{\lambda_{\mathrm{g}}}\right)\right]
$$

et :

$$
E_{2}=A_{2} \exp \left[j\left(\omega t-\varphi_{2}-\frac{2 \pi d_{2}}{\lambda_{\mathrm{g}}}\right)\right]
$$

La vibration résultant de l'addition de ces deux vibrations a pour valeur :

$$
E=E_{1}+E_{2} \text {. }
$$

La détection étant généralement quadratique, cherchons le carré de l'amplitude de cette vibration $\left(E . E^{*}\right)$ :

$I=A_{1}^{2}+A_{2}^{2}+2 A_{1} A_{2} \cos \left(\varphi_{2}-\varphi_{1}-2 \pi \frac{d_{1}-d_{2}}{\lambda_{\mathrm{g}}}\right)$.

Pour avoir une sensibilité maximale, on cherche habituellement à obtenir par le jeu de déphaseurs $\left(^{1}\right.$ ) et d'atténuateurs ( $\left.{ }^{2}\right)$ étalonnés un minimum nul.

Le minimum nul est obtenu lorsque $A_{1}=A_{2}$ et :

$$
\varphi_{2}-\varphi_{1}-2 \pi \frac{d_{1}-d_{2}}{\lambda_{\mathrm{g}}}=(2 k+1) \pi \text {. }
$$

(1) Déphaseur est pris au sens très général de dispositif faisant varier la phase, ce peut être par introduction ou rotation d'une lame, par changement de la longueur électrique, par déplacement de la sonde d'un M.O.S. [1], par la variation de propriétés magnétiques [4], etc.

(2) Atténuateur est également pris au sens très général. 
La mesure se fait en deux temps : on réalise d'abord le minimum nul en l'absence du phénomène à étudier (à vide), puis en présence du phénomène. Partant des variations des atténuateurs et des déphaseurs, on obtient les valeurs de l'absorption et du déphasage produits par le phénomène.

Considérons maintenant le cas d'un interféromètre à trois ondes. Les ondes :

$$
\begin{aligned}
& E_{1}=A_{1} \exp \left[j\left(\omega t-\varphi_{1}-2 \pi \frac{d_{1}}{\lambda_{\mathrm{g}}}\right)\right] \\
& E_{2}=A_{2} \exp \left[j\left(\omega t-\varphi_{2}-2 \pi \frac{d_{2}}{\lambda_{\mathrm{g}}}\right)\right] \\
& E_{3}=A_{3} \exp \left[j\left(\omega t-\varphi_{3}-2 \pi \frac{d_{3}}{\lambda_{\mathrm{g}}}\right)\right]
\end{aligned}
$$

viennent interférer et donnent une vibration :

$$
E^{\prime}=E_{1}+E_{2}+E_{3}
$$

dont le carré de l'amplitude $I^{\prime}$ a pour valeur :

$$
\begin{aligned}
I^{\prime}=A_{1}^{2}+A_{2}^{2} & +A_{3}^{2} \\
& +2 A_{1} A_{2} \cos \left(\varphi_{2}-\varphi_{1}-2 \pi \frac{d_{1}-d_{2}}{\lambda_{\mathrm{g}}}\right) \\
& +2 A_{1} A_{3} \cos \left(\varphi_{1}-\varphi_{3}-2 \pi \frac{d_{3}-d_{1}}{\lambda_{\mathrm{g}}}\right) \\
& +2 A_{2} A_{3} \cos \left(\varphi_{2}-\varphi_{3}-2 \pi \frac{d_{3}-d_{2}}{\lambda_{\mathrm{g}}}\right) .
\end{aligned}
$$

On n'envisagera pas ici l'utilisation d'une variation connue et importante de l'onde $E_{3}$ (avec $A_{3}$ petit) pour compenser la variation inconnue mais faible de $E_{1}$ ou $E_{2}$, ce qui permet dans certaines conditions ( $A_{3}$ petit) d'augmenter la sensibilité mais sans éviter certaines difficultés provenant, en particulier, des atténuateurs et des déphaseurs.

On limitera cette étude à celle de deux cas particuliers importants qui permettent de mesurer et d'enregistrer sans déplacement et en continu les faibles variations de phase et d'amplitude.

Supposons que les variations de $A_{1}, A_{2}, A_{3}, \varphi_{1}, \varphi_{2}, \varphi_{3}$ soient faibles. La différentielle de $I^{\prime}$ a pour valeur : $\mathrm{d} I^{\prime}=2 A_{1} \mathrm{~d} A_{1}+2 A_{2} \mathrm{~d} A_{2}+2 A_{3} \mathrm{~d} A_{3}$

$$
\begin{aligned}
& +2\left(A_{1} \mathrm{~d} A_{2}+A_{2} \mathrm{~d} A_{1}\right) \cos \left(\varphi_{2}-\varphi_{1}-2 \pi \frac{d_{1}-d_{2}}{\lambda_{\mathrm{g}}}\right) \\
& -2 A_{1} A_{2} \sin \left(\varphi_{2}-\varphi_{1}-2 \pi \frac{d_{1}-d_{2}}{\lambda_{\mathrm{g}}}\right)\left(\mathrm{d} \varphi_{2}-\mathrm{d} \varphi_{1}\right) \\
& +2\left(A_{1} \mathrm{~d} A_{3}+A_{3} \mathrm{~d} A_{1}\right) \cos \left(\varphi_{1}-\varphi_{3}-2 \pi \frac{\mathrm{d}_{3}-\mathrm{d}_{1}}{\lambda_{\mathrm{g}}}\right) \\
& -2 A_{1} A_{3} \sin \left(\left(\varphi_{1}-\varphi_{3}-2 \pi \frac{d_{3}-d_{1}}{\lambda_{\mathrm{g}}}\right)\left(\mathrm{d} \varphi_{1}-\mathrm{d} \varphi_{3}\right)\right. \\
& +2\left(A_{2} \mathrm{~d} A_{3}+A_{3} \mathrm{~d} A_{2}\right) \cos \left(\varphi_{2}-\varphi_{3}-2 \pi \frac{d_{3}-d_{2}}{\lambda_{\mathrm{g}}}\right) \\
& -2 A_{2} A_{3} \sin \left(\varphi_{2}-\varphi_{3}-2 \pi \frac{d_{3}-d_{2}}{\lambda_{\mathrm{g}}}\right)\left(\mathrm{d} \varphi_{2}-\mathrm{d} \varphi_{3}\right) .
\end{aligned}
$$

Telle est la variation de l'intensité observée dans le cas général en supposant l'onde initiale rigoureusement monochromatique. Pour pouvoir utiliser ce résultat, il faut faire des hypothèses simplificatrices.

1 er GAS PARTiGulier. - Supposons que :

$$
\mathrm{d} A_{1}=\mathrm{d} \varphi_{1}=\mathrm{d} A_{3}=\mathrm{d} \varphi_{3}=0 .
$$

Les ondes $E_{1}$ et $E_{3}$ ne varient pas au cours de l'étude, seule l'onde $E_{2}$ varie en phase et en amplitude, le phénomène à étudier est dans la branche 2 . Posons :

$$
A_{3}=\frac{A_{1}}{n} \quad \text { et } \quad \varphi_{3}=\varphi_{1}-2 \pi \frac{d_{3}-d_{1}}{\lambda_{\mathrm{g}}}-\gamma
$$

$\mathrm{d} I^{\prime}$ prend alors la valeur $\mathrm{d}\left(I^{\prime}\right)_{1}$ :

$$
\begin{aligned}
\mathrm{d}\left(I^{\prime}\right)_{1} & =2 A_{2} \mathrm{~d} A_{2} \\
& +2 A_{1} \mathrm{~d} A_{2} \cos \left(\varphi_{2}-\varphi_{1}-2 \pi \frac{d_{1}-d_{2}}{\lambda_{\mathrm{g}}}\right) \\
& -2 A_{1} A_{2} \sin \left(\varphi_{2}-\varphi_{1}-2 \pi \frac{d_{1}-d_{2}}{\lambda_{\mathrm{g}}}\right) \mathrm{d} \varphi_{2} \\
& +2 \frac{A_{1}}{n} \mathrm{~d} A_{2} \cos \left(\varphi_{2}-\varphi_{1}-2 \pi \frac{d_{1}-d_{2}}{\lambda_{\mathrm{g}}}+\gamma\right) \\
& -2 A_{2} \frac{A_{1}}{n} \sin \left(\varphi_{2}-\varphi_{1}-2 \pi \frac{d_{1}-d_{2}}{\lambda_{\mathrm{g}}}+\gamma\right) \mathrm{d} \varphi_{2} .
\end{aligned}
$$

Supposons que l'appareil soit réglé à vide de manière telle que $A_{1}=A_{2}$ et plaçons-nous dans des conditions telles que :

$$
\varphi_{2}-\varphi_{1}-2 \pi \frac{d_{1}-d_{2}}{\lambda_{\mathrm{g}}}=(2 k+1) \pi
$$

c'est-à-dire dans les conditions nécessaires pour obtenir un minimum nul dans le cas d'un interféromètre à deux ondes (il suffit de faire (à vide) $n=\infty$ pour déterminer ces conditions) :

$$
\mathrm{d}\left(I^{\prime}\right)_{1}=-\frac{2 A_{1}}{n} \mathrm{~d} A_{2} \cos \gamma+2 \frac{A_{1}^{2}}{n} \mathrm{~d} \varphi_{2} \sin \gamma .
$$

Pour les valeurs particulières de $\gamma$, le tableau I donne les valeurs correspondantes de $\mathrm{d}\left(I^{\prime}\right)_{1}$.

TABLEAU I

$$
\begin{array}{cc}
\stackrel{\gamma}{-} & -\frac{\mathrm{d}\left(I^{\prime}\right)_{1}}{n} \\
0 & -\frac{2 A_{1}}{n} \mathrm{~d} A_{2} \\
\pi / 2 & 2 \frac{A_{1}^{2}}{n} \mathrm{~d} \varphi_{2} \\
\pi & \frac{2 A_{1}}{n} \mathrm{~d} A_{2} \\
-\pi / 2 & -\frac{2 A_{1}^{2}}{n} \mathrm{~d} \varphi_{2}
\end{array}
$$


Donc, si l'onde 3 est en phase ou en opposition de phase avec l'onde 1 , on obtient une variation d'intensité dépendant de la variation d'amplitude $\mathrm{d} A_{2}$, mais indépendante de la variation de phase $\mathrm{d} \varphi_{2}$. On peut donc mesurer $\mathrm{d} A_{2}$.

Lorsqu'au contraire l'onde 3 est en quadrature avance ou retard avec l'onde 1 , la variation d'intensité est indépendante de la variation d'amplitude $\mathrm{d} A_{2}$ et ne dépend que de la variation de phase $\mathrm{d} \varphi_{2}$ que nous pouvons ainsi mesurer.

L'interféromètre à trois ondes permet donc, avec ce réglage particulier, de mesurer indépendamment les faibles variations de phase et d'amplitude et d'en faire l'enregistrement en continu. Avant d'en donner un exemple de réalisation pratique, nous allons envisager un second cas particulier important.

$2^{\text {e }}$ CAS PARticulier. - Supposons $\mathrm{d} A_{3}=\mathrm{d} \varphi_{3}=0$; donc il y a un phénomène dans chacune des branches 1 et 2 , ces phénomènes provoquent de faibles variations des phases et des amplitudes. La variation d'intensité prend alors la forme $\mathrm{d}\left(I^{\prime}\right)_{2}$ :

$$
\begin{aligned}
& \mathrm{d}\left(I^{\prime}\right)_{2}=2 A_{1} \mathrm{~d} A_{1}+2 A_{2} \mathrm{~d} A_{2}+2\left(A_{1} \mathrm{~d} A_{2}+A_{2} \mathrm{~d} A_{1}\right) \\
& \cos \left(\varphi_{2}-\varphi_{1}-2 \pi \frac{d_{1}-d_{2}}{\lambda_{\mathrm{g}}}\right) \\
& -2 A_{1} A_{2} \sin \left(\varphi_{2}-\varphi_{1}-2 \pi \frac{d_{1}-d_{2}}{\lambda_{\mathrm{g}}}\right)\left(\mathrm{d} \varphi_{2}-\mathrm{d} \varphi_{1}\right) \\
& +2 A_{3} \mathrm{~d} A_{1} \cos \left(\varphi_{1}-\varphi_{3}-2 \pi \frac{d_{3}-d_{1}}{\lambda_{\mathrm{g}}}\right) \\
& -2 A_{1} A_{3} \sin \left(\varphi_{1}-\varphi_{3}-2 \pi \frac{d_{3}-d_{1}}{\lambda_{\mathrm{g}}}\right) \mathrm{d} \varphi_{1} \\
& +2 A_{3} \mathrm{~d} A_{2} \cos \left(\varphi_{2}-\varphi_{3}-2 \pi \frac{d_{3}-d_{2}}{\lambda_{\mathrm{g}}}\right) \\
& -2 A_{2} A_{3} \sin \left(\varphi_{2}-\varphi_{3}-2 \pi \frac{d_{3}-d_{2}}{\lambda_{\mathrm{g}}}\right) \mathrm{d} \varphi_{2} .
\end{aligned}
$$

Si, l'appareil étant à vide, on pose :

$$
A_{3}=\frac{A_{1}}{n} \quad \text { et } \quad \varphi_{3}=\varphi_{1}-2 \pi \frac{d_{3}-d_{1}}{\lambda_{\mathrm{g}}}-\gamma
$$

et si le réglage à vide est fait de telle sorte que :

$$
A_{1}=A_{2}
$$

(ce qui n'implique pas $\mathrm{d} A_{1}=\mathrm{d} A_{2}$ ) et :

$$
\varphi_{2}-\varphi_{1}-2 \pi \frac{d_{1}-d_{2}}{\lambda_{\mathrm{g}}}=(2 k+1) \pi
$$

(conditions du minimum nul dans le cas d'un interféromètre à deux ondes), on a alors :

$$
\begin{aligned}
\mathrm{d}\left(I^{\prime}\right)_{2}=2 \frac{A_{1}}{n} \mathrm{~d} A_{1} & \cos \gamma-2 \frac{A_{1}^{2}}{n} \sin \gamma \mathrm{d} \varphi_{1} \\
& -2 \frac{A_{1}}{n} \mathrm{~d} A_{2} \cos \gamma+2 \frac{A_{1}^{2}}{n} \sin \gamma \mathrm{d} \varphi_{2}
\end{aligned}
$$

le tableau II donne les valeurs de $\mathrm{d}\left(I^{\prime}\right)_{2}$ pour les valeurs particulières de $\gamma$.
TABLEAU II

$$
\begin{array}{cc}
\stackrel{\gamma}{\gamma} & \underline{\mathrm{d}\left(I^{\prime}\right)_{2}} \\
0 & 2 \frac{A_{1}}{n}\left(\mathrm{~d} A_{1}-\mathrm{d} A_{2}\right) \\
\pi / 2 & 2 \frac{A_{1}^{2}}{n}\left(\mathrm{~d} \varphi_{2}-\mathrm{d} \varphi_{1}\right) \\
\pi & -2 \frac{A_{1}}{n}\left(\mathrm{~d} A_{1}-\mathrm{d} A_{22}\right) \\
-\pi / 2 & -2 \frac{A_{1}^{2}}{n}\left(\mathrm{~d} \varphi_{2}-\mathrm{d} \varphi_{1}\right)
\end{array}
$$

Si l'onde 3 est en phase ou en opposition de phase avec l'onde 1 , on obtient une variation d'intensité dépendant de la différence des variations des amplitudes des ondes 1 et 2 mais indépendante des variations des phases de ces ondes.

Si l'onde 3 est en quadrature avance ou retard avec l'onde 1, la variation d'intensité est fonction de la différence des variations de phases des ondes 1 et 2 , mais indépendante des variations des amplitudes.

L'interféromètre à trois ondes est donc dans ce cas particulier un interféromètre « différentiel » permettant de mesurer indépendamment et d'enregistrer les différences de variations de phases et d'amplitudes produites par deux phénomènes simultanés introduits dans chacune de ses branches.

L'étude qui vient d'être faite est très générale et la réalisation pratique que j'ai réalisée a consisté à ajouter une troisième voie à l'interféromètre mis au point par MM. P. Loudette et F. Picherit [1]. Ce montage a été choisi en raison de sa simplicité de mise en œuvre et des bons résultats qu'il donne, moyennant certaines précautions; mais il est bien évident que le procédé est applicable à tous les interféromètres à deux ondes : c'est l'adjonction d'une troisième onde de phase et d'amplitude réglables servant à la « détection de phase ».

Réalisation pratique. - La figure 1 représente le schéma d'une des réalisations qui en ont été faites au Laboratoire.

La source est constituée par un klystron X 13 Varian immergé dans l'huile. Il est stabilisé par un montage à transistors du type Jung [5] déjà utilisé au Laboratoire [6].

Pour pouvoir utiliser un tosmètre, l'onde est modulée à $1000 \mathrm{~Hz}$ par un modulateur à ferrite, ce qui évite les variations de fréquence qu'introduirait une modulation par signaux rectangulaires sur le réflex du klystron.

Suivant l'étude envisagée, on choisira le premier ou le deuxième cas et on placera dans les rectangles pointillés les éléments permettant d'obtenir, s'il y a lieu, la ou les branches ouvertes nécessaires à l'étude. 


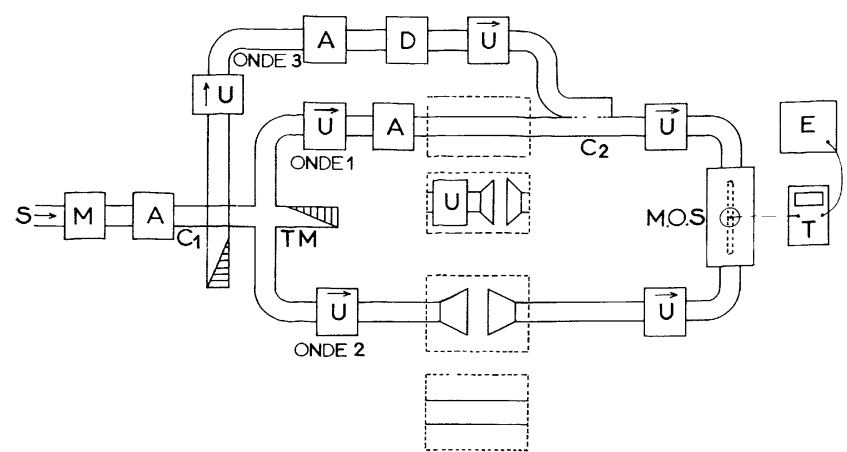

FIG. 1. - S, Source stabilisée ; M, Modulateur ; TM, Té magique; A, Atténuateur tournant; U, Uniligne; $\mathrm{C}_{1}$, Coupleur directif $15 \mathrm{~dB} ; \mathrm{C}_{2}$, Coupleur directif $10 \mathrm{~dB}$; $\mathrm{D}$, Déphaseur à élément tournant; E, Enregistreur ; T, Tosmètre.

Pour éviter les réflexions multiples entre les divers éléments du montage, ceux-ci doivent être adaptés séparément. En particulier, les unilignes doivent être soigneusement adaptés dans le sens direct et dans le sens inverse. Les atténuateurs et les déphaseurs ont été choisis à éléments tournants car d'une part ils introduisent des T.O.S. faibles et sensiblement constants, et d'autre part les déphasages et les atténuations qu'ils apportent respectivement sont réduits, ce qui facilite les réglages. Moyennant quelques précautions, l'expérience montre que l'appareil permet de mesurer et d'enregistrer en continu des variations de phase de quelques secondes et des variations relatives d'amplitude $\left(\mathrm{d} A_{2} / A_{2}\right)$ de l'ordre de $10^{-5}$.

Il convient de remarquer que l'appareil permet l'étude des phénomènes très rapides en supprimant le modulateur et le tosmètre et en remplaçant par exemple l'enregistreur par un oscilloscope avec dispositif photographique.

Projet de Perfectionnement. - L'appareil tel qu'il a été réalisé ne permet pas de mesurer simultanément les variations de phase et d'amplitude. Mais le montage ( $f g .2$ ), qui n'a pas été réalisé encore, permettrait la mesure simultanée (et l'enregistrement des variations de phase et d'amplitude).
L'amélioration de la sensibilité de l'interféromètre à trois ondes, tout au moins dans une certaine mesure, ne pose aucun problème technique mais un problème de matériel. En effet, le calcul montre que la sensibilité peut être théoriquement augmentée par l'utilisation

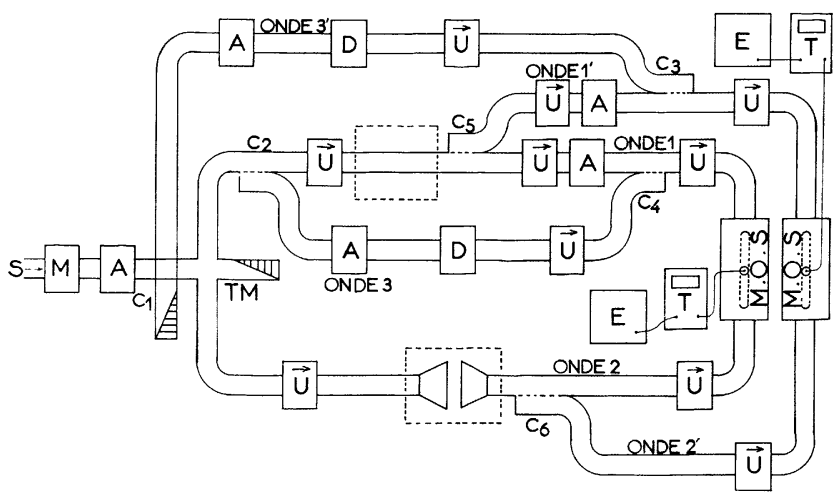

FIG. 2. - S, Source stabilisée ; M, Modulateur ; TM, Té magique; $\mathrm{A}$, Atténuateur tournant; $\mathrm{U}$, Uniligne ; $\mathrm{C}_{1}, \mathrm{C}_{2}$, Coupleurs directifs $15 \mathrm{~dB} ; \mathrm{C}_{5}, \mathrm{C}_{6}$, Coupleurs directifs $3 \mathrm{~dB} ; \mathrm{C}_{3}, \mathrm{C}_{4}$, Coupleurs directifs $10 \mathrm{~dB}$; $D$, Déphaseur à élément tournant; E, Enregistreur ; $T$, Tosmètre.

d'une source plus puissante et d'un système de détection plus sensible (détection synchrone par exemple), mais avec certaines limitations dues en particulier à la stabilité de la source en fréquence et en puissance, à son spectre de bruit et au bruit de fond du système de détection.

Conclusion. - Dans l'immédiat, le montage de la figure 1 est destiné à l'étude des variations de phase et d'amplitude produites par l'action d'un champ magnétique sur des sels paramagnétiques, mais il a été déjà utilisé en polarimétrie hertzienne [7]. De nombreuses autres utilisations peuvent être envisagées : enregistrement en continu des variations de phase et d'amplitude produites par le défilement d'une feuille de papier [3]; variation du taux d'humidité entre l'entrée et la sortie d'une feuille de papier passant dans une machine, études des plasmas, de constantes diélectriques, etc.

\section{BIBLIOGRAPHIE}

[1] LoudetTe: (P.) et Picherit (F.), J. Physique Rad., 1960, 21, 11, $92 \mathrm{~S}$.

[2] Bottreiau (A.) et Marzat (C.), C. R. Acad. Sc. Pavis, 1964, 259, 758.

[3] Bottreau (A.) et Marzat (C.). Onde élecivique, 1965, 457, 4, 485 .
[4] Constantinou (E.) et Counes, Rev. Phys. Appl., mars $1968,3,21$.

[5] Jung (P.), J. Scientific Instrument, 1960, 37, 372.

[6] Sardos (R.), Thèse, Bordeaux, 1965.

[7] Sardos (R.), C. R. Acad. Sc. Pavis, 1966, 263, 38. 